\title{
Consequences of AMPK activation
}

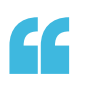

...human

carriers of the

homologous

mutation ...

also exhibit

increased

adiposity...
An activating mutation in the gene that encodes the $\gamma 2$ subunit of AMPK causes obesity in mice, according to new research.

Therapeutic activation of AMPK has been proposed as a strategy to treat systemic metabolic diseases. As such, a team of researchers led by Arash Yavari and Houman Ashrafian decided to investigate the long-term consequences of AMPK activation.

To create a mouse model of increased AMPK activation, the team introduced an Arg299Gln mutation

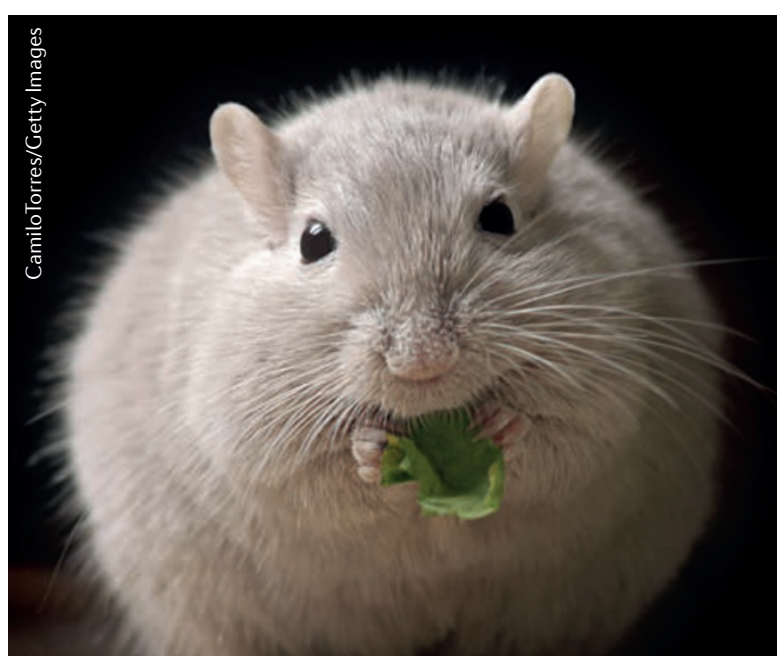

into Prkag2 (which encodes the murine $\gamma 2$ subunit); the resulting mice were termed R299Q $\gamma 2$ mice. The $\gamma 2$-specific AMPK activity of these mice was almost three times greater than that of wild-type mice.

At 40 weeks old, homozygous R299Q $\gamma 2$ mice had greater fat mass and frank hepatic steatosis compared with wild-type mice, as well as an increase in plasma levels of proinflammatory cytokines. These mice also had glucose intolerance and reduced insulin sensitivity, which are hallmarks of obesity.

Pair-feeding experiments with R299Q $\gamma 2$ and wild-type mice revealed that hyperphagia was the main contributor to the obesity of R299Q $\gamma 2$ mice. Further investigation demonstrated that the hyperphagia of these mice was dependent on increased ghrelin receptor signalling, which lowered the threshold for feeding.

Mice carrying this mutation also had impaired $\beta$-cell function, with reduced glucose-stimulated insulin secretion. The researchers showed that key functional islet genes, including those that encode insulin and glucokinase (Ins 1, Ins2 and
$G c k$ ), were downregulated in islets from R299Q $\gamma 2$ mice, while several genes whose expression is normally selectively repressed in mature $\beta$ cells were upregulated.

Importantly, heterozygous human carriers of the homologous mutation (an Arg302Gln missense mutation in PRKAG2) also exhibited increased adiposity, an increase in plasma markers of steatosis and reduced basal $\beta$-cell function with elevated $\mathrm{HbA}_{1 \mathrm{c}}$.

"Our findings led us to conclude that long-term AMPK activation throughout all tissues can exert adverse metabolic consequences (particularly increased appetite and reduced $\beta$-cell function), which we suggest should be taken into account in pharmacological approaches that seek to chronically activate AMPK systemically," conclude Yavari and Ashrafian. "Nonspecific, generalized long-term AMPK activation, without reference to subunit composition or individual tissue effects, might result in harm rather than benefit."

Claire Greenhill

ORIGINAL ARTICLE Yavari, A. et al. Chronic activation of $\gamma 2$ AMPK induces obesity and reduces $\beta$ cell function. Cell Metab. http://dx.doi. org/10.1016/j.cmet.2016.04.003 (2016) 